\title{
Tissue harmonic imaging
}

\author{
Talat Uppal
}

Northern Beaches Maternity, Manly Hospital, Manly, New South Wales 2065, Australia. Correspondence to author via ASUM. Email authors@asum.com.au

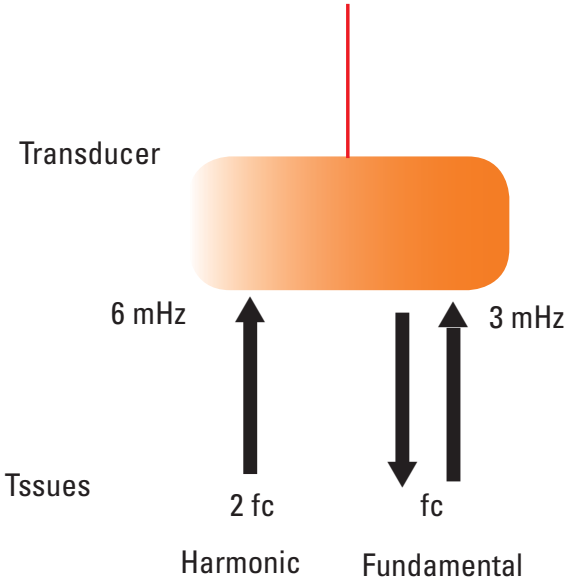

Fig. 1: Fundamental echoes must pass through the body twice, whereas harmonics originate in the body and therefore are less subject to distortion from non-linear propagation. Higher frequency multiples of the interrogating frequency are called second $(2 \mathrm{fc})$ or third $(3 \mathrm{fc})$ harmonic frequencies.

Facebook quote: "Learning about harmonics on a guitar is more fun than harmonics of an ultrasound machine" :(

Harmonics have much significance in music.

A musical note has three characteristics.

1 Pitch (or frequency)

2 Loudness

3 Quality (or tone).

Quality is the audible difference heard between two musical notes of the same pitch and loudness.

Hence, a piano based C-note does not sound the same as a C-note played on a guitar, due to the existence of harmonic frequencies. The same note from various instruments has different qualities because the sounds are not pure notes i.e. of one frequency and these integral multiples of the fundamental frequency (overtones) give an instrument its characteristic sound.

In an ultrasound context, tissue harmonic imaging is a signal processing technique also termed native harmonic imaging. An ultrasound beam insonates body tissues and generates such harmonic waves from nonlinear distortion during the transmit phase of the pulse-echo cycle.

This imaging method was discovered by accident in USA when scientists working on the development of microbubbles for contrast in echocardiography found that a tissue image was still created when the receiver was tuned to receive at twice the transmitted frequency. This meant harmonics frequencies arose from soft tissue even without the injection of microbubble contrast agents. In fact some of the features of the image were superior to the fundamental image.

The ultrasonic pulse gets altered with time as it traverses the tissues with non-linear motion. The peaks within the pulse waveform move faster than the troughs because the

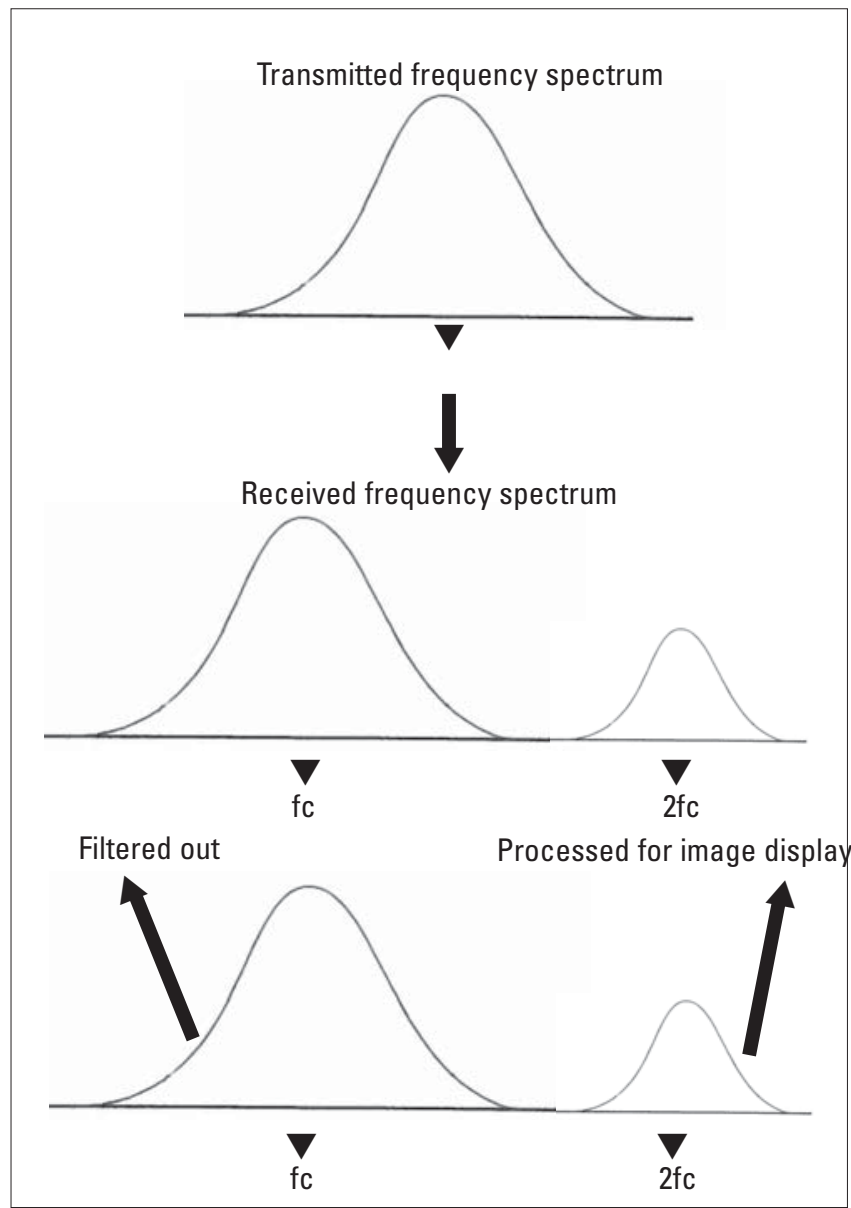

Fig. 2: Tissue harmonic images are obtained by collecting harmonic signals that are tissue generated and filtering out the fundamental echo signals that are transducer generated resulting in crisper images.

propagation speed is higher in compressed regions of tissue than in the areas which are expanded by the passing pressure wave. The degree of such acoustic signal distortion in tissue depends on the amplitude of the emitted pulse and the distance it has travelled in the tissue.

For example, a $3.0 \mathrm{MHz}$ transducer transmits a band of frequencies centered on the $3.0 \mathrm{MHz}$ frequency. In traditional imaging, when tissue is insonated with a band of fundamental frequencies centered at $3.0 \mathrm{MHz}$, most of the returning echoes to the transducer will be linked to the same $3 \mathrm{MHz}$ frequency band. Harmonics are multiples of the fundamental frequency, hence transmitting a band of frequencies centered at $3 \mathrm{MHz}$ will result in the production of harmonic frequency bands centered at $6 \mathrm{MHz}, 9 \mathrm{MHz}$, $12 \mathrm{MHz}$, etc.

\section{Harmonics production}

Two different processes can be utilised to create harmonic signals: 


\section{Benefits of harmonic imaging}

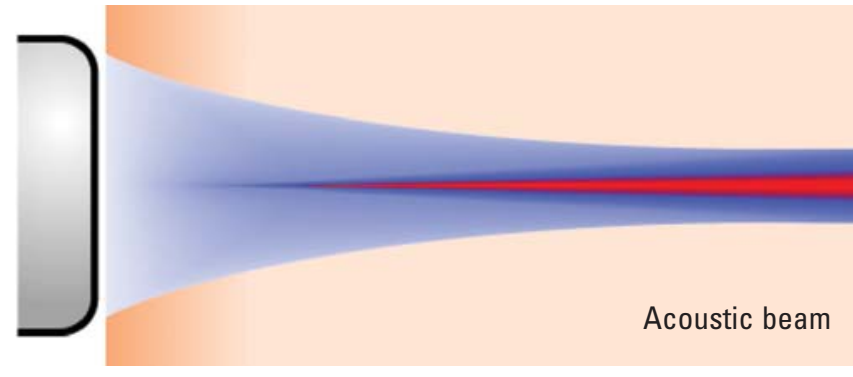

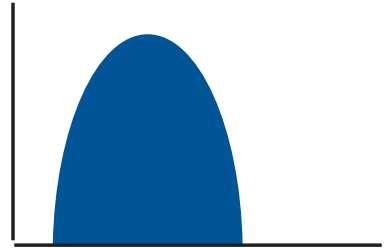

Transmitted echo

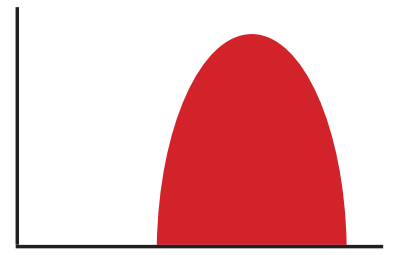

Received signals (filtered version)
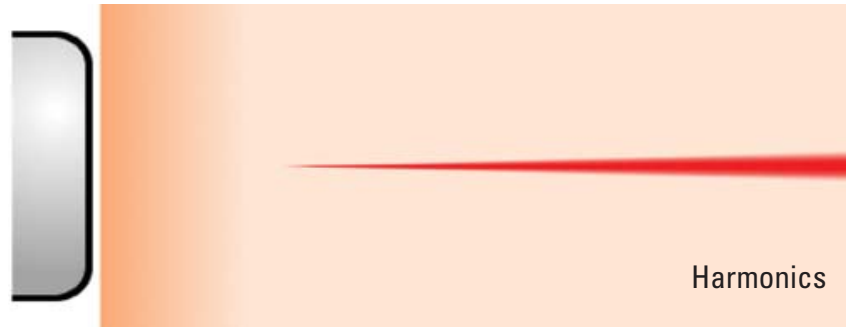

Note: Central position in beam and narrower profile.

1 By using filters to screen out the fundamental from the harmonic signals (as depicted in Fig. 2)

2 By using two simultaneous pulses with a $180^{\circ}$ phase difference (pulse inversion imaging).

\section{Contrast harmonic imaging}

Harmonic imaging can involve injection of contrast agent with the appropriate microbubbles and then scanning the region of interest at a given transmit frequency and tuning the receiver to listen at twice that value. For example, transmit at $3 \mathrm{Mhz}$ and receive at $6 \mathrm{Mhz}$.

The result is that the backscattered echoes from resonant bubbles contain harmonics

Harmonic imaging can be performed in B mode and Doppler mode.

Not all microbubble contrast agents are suitable for harmonic imaging - the bubble size is important, as is the surrounding shell, which must be flexible to allow resonance to occur.

The strongest echoes recorded will be those from the regions where there is contrast like the blood vessels. Echoes from the tissues surrounding the vessels, being composed of the fundamental frequency rather than the second harmonic, produce a much lower response in the receiver and therefore have a reduced brightness in the display ( in comparison to their appearance in a standard B mode imaging)

The returning increased frequency harmonic signal has to travel one direction only, towards the probe. The advantages of high frequency imaging and the one-way travel effect are reduced reverberation, beam aberration, and side

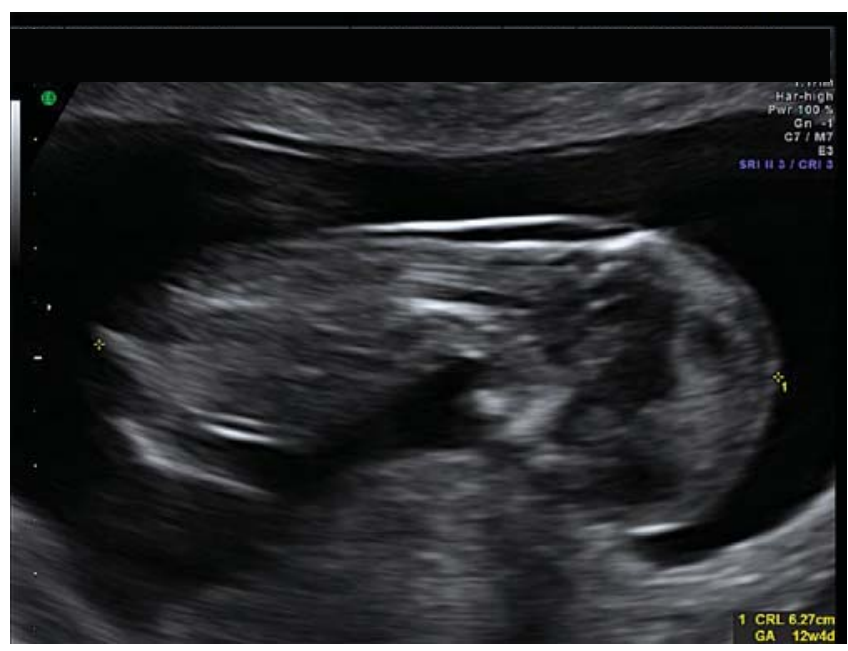

Fig. 3: First trimester scan with harmonics "on".

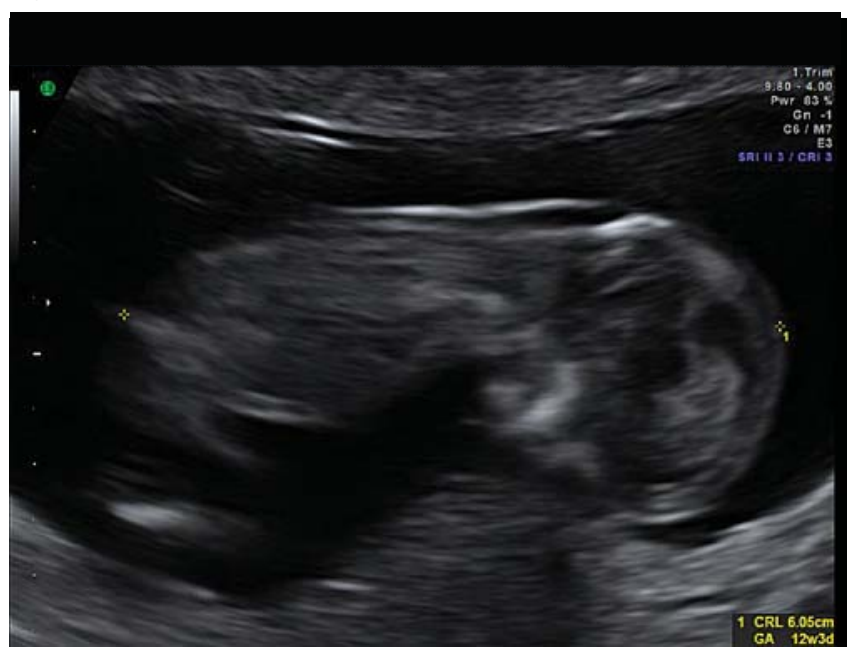

Fig. 4: First trimester scan with harmonics "off".

lobes plus improved resolution and cystic clearing.

- Improved axial resolution due to higher frequencies

- Better lateral resolution due to narrower beams

- Decreased noise from side lobes improves signal-tonoise ratios and reduces artifacts

- Improved range penetration

- Better contrast resolution

- Harmonic imaging can be particularly useful for depicting cystic lesions and those containing echogenic tissues such as fat, calcium, or air

- In patients with a body mass index of 30 or more, harmonic imaging can lead to better lesion visibility and confidence of diagnosis

- Improved imaging of the liver, gallbladder, pancreas, pelvis, kidneys, and retroperitoneal lymph nodes

- Additionally patients who were imaged poorly with fundamental frequency ultrasound could be examined by second harmonic imaging.

Disadvantage of less penetration, more shadowing and could confuse with respect to possible calculi.

\section{Summary}

Ultrasound wave travels faster in compressive phase and slower during rarefaction (reduced particle density).

This nonlinear propagation changes a part of the beams energy to harmonic frequencies.

The second harmonic has a frequency which is twice the fundamental frequency and contains most of the har- 
monic energy.

Harmonics are not produced in the superficial part of tissue.

Harmonics are most prominent in the central section of the transmitted beam. Hence lateral resolution is improved in harmonic mode

Reduced Image artefacts like reverberations, side and grating lobes

Improved contrast resolution as less "noise" and clutter (better signal to noise ratio).

\section{References}

1 Kollman C, New sonographic techniques for harmonic imaging underlying physical principles. Eur J Radiol 64 (2007) 164-72.

2 Kremkau F. Diagnostic ultrasound, Principles and instrumentation. Heidelberg: Springer-Verlag; 2006.

3 Zagzebski J. Essentials of ultrasound physics. Amsterdam: Elsevier; 1996.
4 Hedrick W, Hykes D, Starchman D. Ultrasound Physics and Instrumentation. St Louis; Mosby: 2005.

5 Gent R. Applied physics and technology of diagnostic ultrasound. Prospect; Milner Publishing: 1997.

6 Shapiro RS, Wagreich J, Parsons RB, et al. Tissue harmonic imaging sonography: evaluation of image quality compared with conventional sonography. AJR 1998; 171: 1203-6.

7 Hann LE, Bach AM, Cramer LD, et al. Hepatic sonography: comparison of tissue harmonic and standard sonography techniques. AJR 1999; 173: 201-6.

8 Tranquart F, Grenier N, Eder V, Pourcelot L. Clinical use of ultrasound tissue harmonic imaging. Ultrasound Med Biol 1999; 6: 889-4.

9 Smith L, Perron A, Perisco A, et al. Understanding the Advanced signal processing technique of real-time adaptive filters. Journal of Diagnostic Medical Sonography 2009; 25: 145-60.

10 Mesurolle B, Helou T, et al. Enhancing image quality using advance signal processing techniques. Journal of diagnostic Medical Sonography 2008; 24: 72-81

If you found this article to be educational you may claim MOSIPP Points in Category 1 at the rate of .25 points per fifteen minutes of reading time. 